\title{
DESIGN OF RADIALLY FOCUSED UNIFORM X-RAY SOURCE
}

\author{
Young-hwan Kim ${ }^{*}$, Hyeok-jung Kwon, Yong-hwan Kim, Han-sung Kim, Sang-ho Kim, \\ Kang-ok Lee and Kie-hyung Chung \\ Department of Nuclear Engineering, Seoul National University, Seoul, 151-742, Korea
}

\begin{abstract}
The $10 \mathrm{MeV}, 100 \mathrm{~kW} \mathrm{CW}$ electron accelerator (FANTRON I) is being developed in Department of Nuclear Engineering, Seoul National University (SNU) [1]. The X-ray generated by the accelerated electron beam will be used in sterilizing the agricultural, forest and aquatic products. For the effective irradiation and the safety of the irradiated products, the uniform irradiation is needed. The designed target is mainly featured by the radially focused uniform X-ray. The diameter of irradiation hole is $0.75 \mathrm{~m}$. And the generated $\mathrm{X}$-ray is emitted toward the center of the irradiation hole with uniform distribution along the circumference. To generate the radially focused uniform X-ray, the accelerated electron beam must be focused radially on the target that is the outer shell of the irradiation hole with high uniformity. Additionally the electron beam must be injected in the normal direction on the surface of target because the intensity of electron incident direction is dominant in the angular distribution of bremsstrahlung intensity of the electrons, which are accelerated up to $10 \mathrm{MeV}$. The basic principle of targetary is deflecting electron beam with proper magnetic field configuration. To find such configuration a series of numerical analyses are performed. This paper includes the requirements and design results of the radially focused uniform X-ray source.
\end{abstract}

\section{INTRODUCTION}

The uniform irradiation is needed to guarantee the safety of the irradiated products and the irradiation performance. Several methods are suggested to increase the radiation dose uniformity [2]. The radially focused uniform X-ray source is composed of scanning magnet, field shaping system and cylindrical irradiation hole. Fig. 1 shows the layout of target system. The $10 \mathrm{MeV}$ electron beam scanned to proper angle is deflected along the optimized trajectories during passing the field shaping system and then collide against the target in the normal direction with azimuthally uniform distribution. The design process comprises three stages.

- Optimization of the electron trajectory

- Calculation of the magnetic field distribution

- Optimization of the magnet pole profile

The target system with 0.75 m-diameter irradiation hole

Email: hessee@paragon.snu.ac.kr

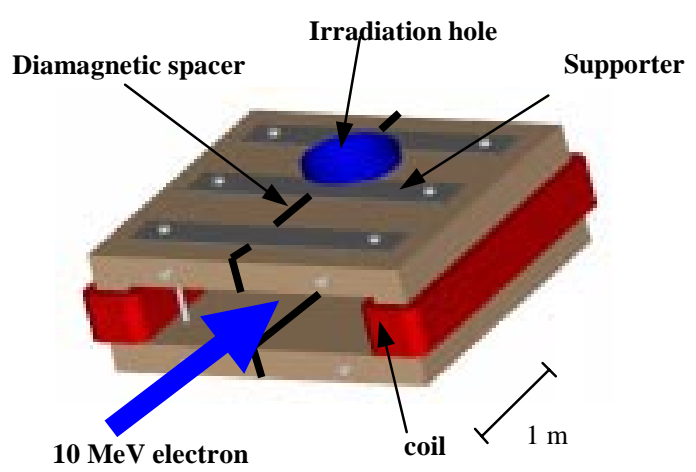

Figure 1. Layout of Target System

is designed to establish and verify the design procedure.

\section{ELECTRON TRAJECTORY OPTIMIZATION}

The deflected electron beam with proper angle after passing the scanning magnet should follow the trajectory along which they collide against the target in the normal direction. The Hermite curve, which is a kind of parametric cubic curve, is suggested as the electron trajectory. The Hermite curve is generated on the base of four control vectors composed of two location vectors and two tangent vectors [3]. The principle of curve generation is described in Eq. (1) and Fig. 2.

$$
\begin{aligned}
\vec{Q}(t) & =\left(2 t^{3}-3 t^{2}+1\right) \vec{P}_{1}+\left(3 t^{2}-2 t^{3}\right) \vec{P}_{4} \\
& +\left(t^{3}-2 t^{2}+1\right) \vec{R}_{1}+\left(t^{3}-t^{2}\right) \vec{R}_{4} \quad(0 \leq t \leq 1)
\end{aligned}
$$

During the optimization, the magnetic field gradients corresponding to each trajectory are minimized and inflection point where the concavity of the curve changes is avoided. The optimized electron trajectories are shown in Fig. 3.

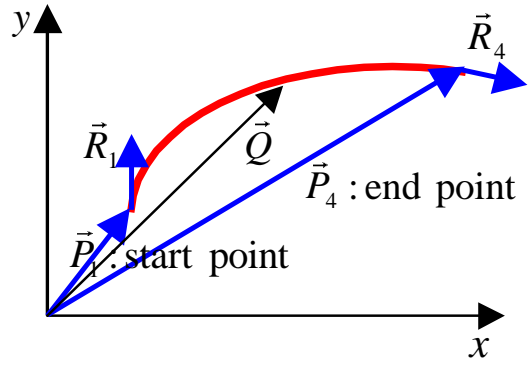

Figure 2. The Principle of Hermite Curve Generation 


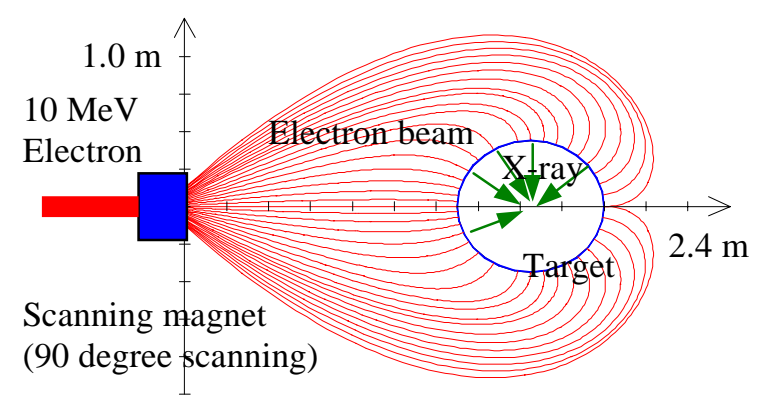

Figure 3. The Optimized Electron Beam Trajectories

\section{MAGNETIC FIELD DISTRIBUTION}

Magnetic field distribution is determined such that electron beam may be deflected along the optimized trajectory. The relativistic equation of motion is used in calculating the field distribution. Fig. 4 shows the magnetic field distribution.

\section{MAGNET POLE PROFILE OPTIMIZATION}

Magnet pole profile design is intrinsically the nonlinear reverse magnetostatic problem. The main approaches are classified into two cases, which are the numerical analyses of Poisson's equation with iteration processes and the analytic approaches. For linear problem, in the case that the magnetic field strength is so low that the saturation effects of iron core do not have any effect on field distribution, several kinds of analytic solutions are suggested [4].

Concerning the result of calculated magnetic field distribution, the maximum field strength is below about $0.05 \mathrm{Ts}$. So the analytic methods are adopted for magnet pole profile design. Three kinds of analytic methods are performed.

- $\quad$ Finite difference method

- Boundary integral method

- Downward continuation method

\subsection{Finite Difference Method}

Magnetostatic problem, in the case that the source free and isotropic region is considered, is solved using magnetic scalar potential in the similar way to electrostatic case. For the linear problem, the pole surface coincides with equipotential surface [4]. The Laplace equation and boundary condition are used in finding the equipotential surface.

$$
\begin{aligned}
& \frac{\partial^{2} \Phi}{\partial x^{2}}+\frac{\partial^{2} \Phi}{\partial y^{2}}+\frac{\partial^{2} \Phi}{\partial z^{2}}=0 \\
& \frac{\partial \Phi}{\partial z}=B_{Z}(x, y), \text { on the midplane }(\mathrm{z}=0) \\
& \quad \Phi: \text { magnetic scalar potential }
\end{aligned}
$$

The equipotential surface obtained from equation (2) and (3) with proper boundary conditions is shown in Fig. 5.

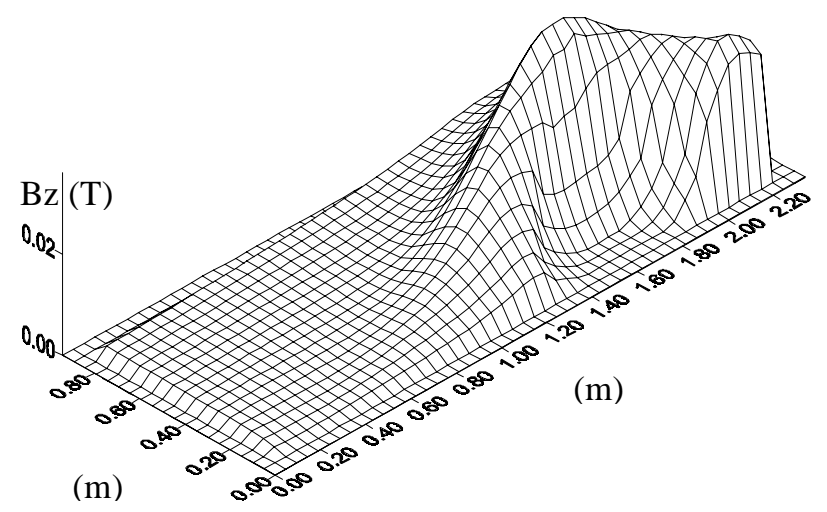

Figure 4. The Magnetic Field Distribution on the midplane of the Magnet

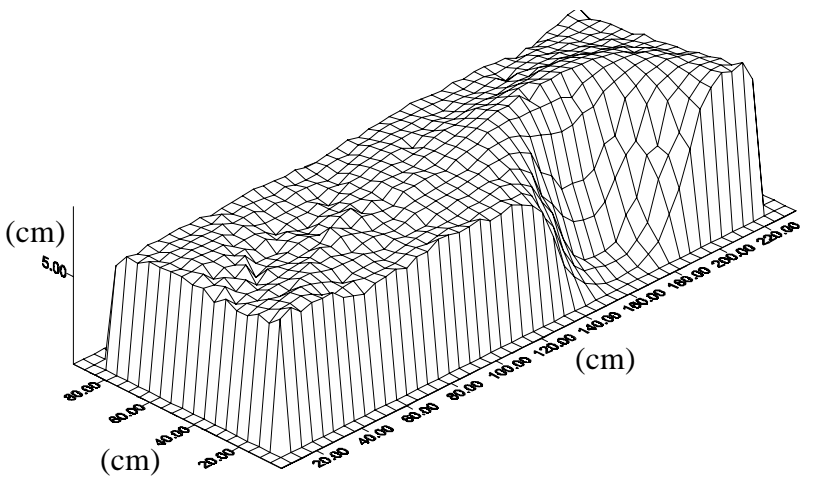

Figure 5. The Equipotential Surface Calculated using Finite Difference method

\subsection{Boundary Integral Method}

The boundary integral method is the approach that the set of points $Q(l, m, z(l, m))$ can be determined so that the performance criterion function $\mathrm{F}$ may be minimized [5]. The magnetic field distribution at points $P(i, j, 0)$ is determined by the set of points $Q(l, m, z(l, m))$ as shown in Fig. 6. And the performance criterion function is :

$$
F=\sum_{i} \sum_{j}\left[\frac{B(i, j, 0)-B_{0}(i, j, 0)}{B_{0}(i, j, 0)}\right]^{2}
$$

$\left(B(i, j, 0)\right.$ : calculated field, $B_{0}(i, j, 0)$ : reference field ) The calculated pole profile is shown in Fig. 7.

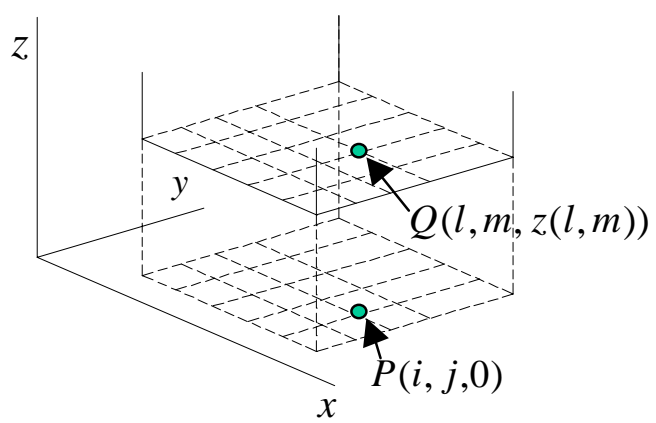

Figure 6. The Boundary Integral Method 


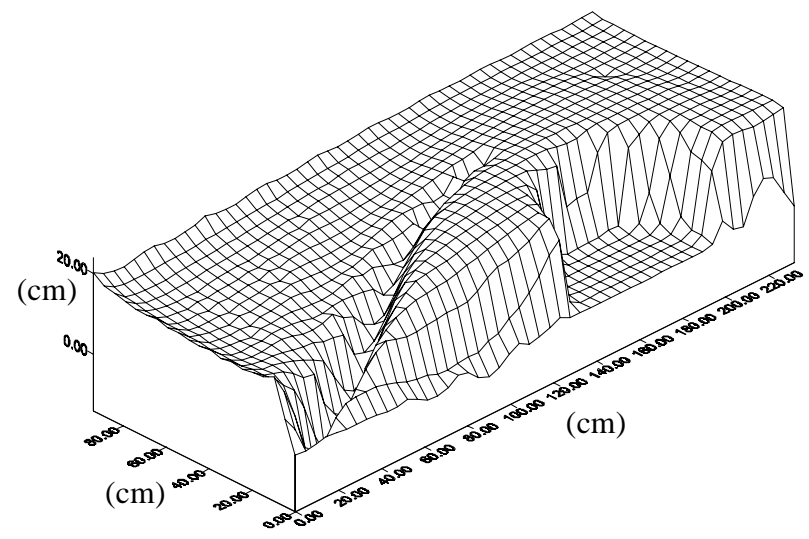

Figure 7. The Magnet Pole Profile Obtained using Boundary Integral Method

\subsection{Downward Continuation Method}

In geophysics, downward continuation method is usually used in estimating the shape of geomagnetic source from the measured magnetic field distribution [6]. This approach can be used to calculate the magnetic potential distribution toward the magnet pole from the midplane magnetic field distribution. The basic principles are Green's third identity (5) and Fourier transformation (6). Green's third identity :

$$
U(P)=\frac{1}{4 \pi} \int_{S}\left(\frac{1}{r} \frac{\partial U}{\partial n}-U \frac{\partial}{\partial n} \frac{1}{r}\right) d S
$$

$U(P)$ : harmonic function at point $P$

$n \quad$ : normal unit vector of the surface $S$

$r \quad$ : distance from point $P$ to any point on $S$

Fourier transformation :

$$
\begin{aligned}
& U(x, y, z+\Delta z)= \\
& F^{-1}\left[F[U(x, y, z)] / F\left[\frac{\Delta z}{2 \pi} \frac{1}{\left(x^{2}+y^{2}+\Delta z^{2}\right)^{3 / 2}}\right]\right]
\end{aligned}
$$

With equation (5) and (6) the potential $U$ at elevated position is obtained from the midplane potential. The designed magnet pole profile is shown in Fig. 8.

There are some intrinsic drawbacks in using this approach [7], [8].

- The Fourier transformation is done in discrete manner.

- The analyzed data are not continuous but discrete (sampled).

- The area over which the Fourier transformation is performed is finite.

\section{CONCLUSIONS AND FUTURE WORKS}

According to the design procedure, the trajectory of electron beam is optimized using Hermite curve. The

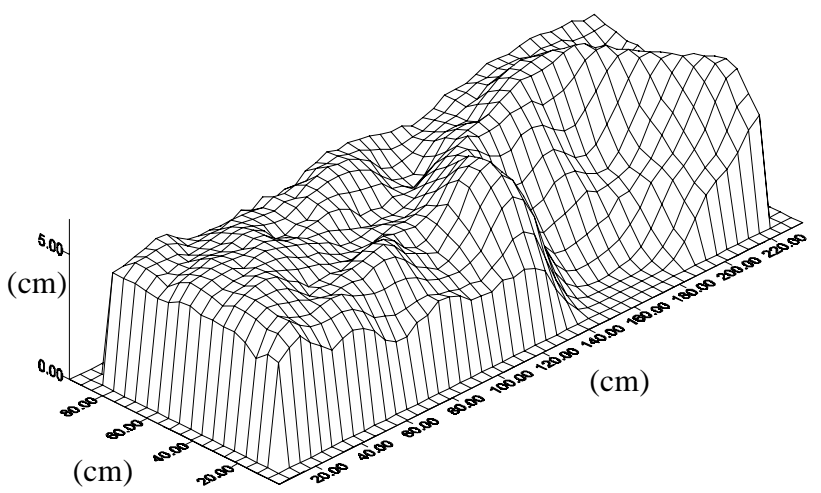

Figure 8. The Magnet Pole Profile Calculated using Downward Continuation Method

magnetic field distribution is determined such that it may deflect the electrons along the optimized trajectory. The magnet pole profile is designed using several analytic approaches. The difference in designed pole profiles between each method may be due to the difference equipotential levels and the intrinsic drawbacks of each method.

For the future works, the estimation of radiation dose uniformity and loss fraction of electron beam for the each pole profile using the $3 \mathrm{~d}$ electron trajectory code are being done. The trim process, which is needed for practical three-dimensional manufacturing, will be done.

\section{REFERENCES}

[1] Hyeok-jung Kwon, Yong-hwan Kim, Young-hwan Kim, Han-sung Kim, Sang-ho Kim, Kang-ok Lee and Kie-hyung Chung, Conceptual Design of $10 \mathrm{MeV}, 100 \mathrm{~kW}$ CW Electron Accelerator for Industrial Application, submitted to PAC '99

[2] A. S. Ivanov, et al., Electromagnetic electron irradiation field shaping system for industrial high voltage accelerators, Proceedings of the EPAC ' 94

[3] James D. Foley, et al., Computer graphics : principles and practice, Addison-Wesley 1996

[4] G. W. Foster, et al. Pole profile optimization of VLHC transmission line magnet, Proceedings of the EPAC '98

[5] A. G. A. M. Armstrong, et al., Automated optimization of magnet design using the boundary integral method, IEEE Transactions on Magnetics, Vol. MAG-18, No. 2, March 1982

[6] Richard J. Blakely, Potential Theory in Gravity and Magnetic Applications, Cambridge University Press, 1995

[7] Y. Ricard, et al., A method to minimize edge effects in twodimensional discrete Fourier transformations, Geophysics, Vol. 53, No. 8, August 1988

[8] L. Cordell, Reconciliation of the discrete and integral Fourier transforms, Geophysics, Vol. 47, No. 2, Feb. 1982 